\title{
Au cœur du piège budgétaire
}

\section{Andreas Gassen}

Dr, Spécialiste en orthopédie, chirurgie des accidents et rhumatologie en pratique privée

Président du comité de I'Union fédérale des médecins conventionnés (Kassenärztliche Bundesvereinigung)

Le budget global dont il est question en Suisse existe depuis 25 ans en Allemagne. Alors qu'il a été introduit pendant une période de pléthore médicale et de forte augmentation des coûts, il est aujourd'hui responsable du fait que $20 \%$ des actes fournis par les cabinets médicaux ne soient pas payés. La colère gronde, surtout depuis que les politiciens exigent des prestations supplémentaires. Les médecins conventionnés allemands s'organisent pour y faire face.

Tout a commencé par une crise. En 1992, l'Allemagne a été confrontée à une augmentation rapide des coûts de la santé, tant dans le secteur ambulatoire qu'hospitalier avec, en même temps, un nombre anormalement élevé de médecins, si bien que même le plus petit hôpital de province recevait plus d'une centaine de candidatures pour un seul poste vacant de médecin-assistant. Dans le secteur ambulatoire en revanche, les médecins spécialistes étaient libres de s'installer pratiquement partout. Le gouvernement de l'époque avait prévu deux mesures pour reprendre en main la question des coûts: premièrement, mettre en place un budget pour les hôpitaux et les cabinets médicaux ambulatoires, tout en introduisant pour les cliniques le principe de concurrence par une privatisation. Deuxièmement, il a suivi la démarche inverse pour les médecins du secteur ambulatoire facturant à la charge de l'assurance-maladie obligatoire. En effet, le nombre de ce que l'on appelle les «Kassensitze» (postes conventionnés) est désormais "planifié» et le nombre de généralistes et de spécialistes autorisés à exercer dans une région est régulé en fonction des besoins. Il en résulte que seul un médecin qui a obtenu un poste par le biais de la planification peut facturer le trai-

\section{Le contrôle de l'économicité, une épée de Damoclès}

Un médecin peut tout à faire subir un contrôle d'économicité même s'il respecte le budget et que ses prestations supplémentaires ne sont pas ou très peu rémunérées. Dans le pire des cas, ce contrôle peut se terminer par la restitution d'honoraires déjà perçus - les fameuses actions récursoires. Les cabinets peuvent être amenés à justifier en détail, pour des cas déjà anciens, pourquoi ils ont traité des patients alors qu'ils avaient déjà dépassé la limite budgétaire fixée pour leur cabinet. S'ils arrivent à prouver les spécificités de leur patientèle ou à fournir des explications au cas par cas, le dépassement budgétaire est toléré. Dans le cas contraire, le remboursement peut atteindre des sommes à six chiffres.

La plupart des associations de médecins conventionnés s'en tiennent au principe selon lequel il vaut mieux conseiller que subir une action récursoire. Elles s'adressent aux cabinets problématiques et leur indiquent comment éviter les dépassements. Les différentes étapes d'une action récursoire ne sont enclenchées qu'en cas de problème répété. Même s'il s'agit d'une épée de Damoclès qui risque de frapper les cabinets médicaux, les actions récursoires ont rarement lieu. tement des patients affiliés à une assurance publique soit $90 \%$ de la population - à la charge de leur assurancemaladie.

Deux verrous ont donc été introduits dans le système: le plafonnement des moyens financiers mis à disposition et une limitation du nombre de médecins autorisés à facturer des prestations ambulatoires. La budgétisation (le budget global) a donc renversé le modèle existant: ce n'était plus les recettes qui devaient couvrir les dépenses mais les dépenses qui ne devaient plus dépasser les recettes.

\section{Le cœur du problème: un budget limité pour un droit illimité aux prestations}

Fixer un plafond budgétaire et limiter le nombre de médecins est une approche plutôt malhabile pour répondre à la dynamique d'une demande de prestations en hausse, liée à une augmentation des coûts. Pour déterminer les ressources financières, des mécanismes ont été introduits afin d'adapter le volume des prestations sur la base de critères démographiques et de mesures de morbidité. Lors de la planification des postes de médecins, il est aussi possible de tenir compte des spécificités régionales. Les deux volets se négocient en Allemagne grâce à ce que l'on appelle l'autogestion commune, c'est-à-dire au travers de discussions entre les associations de médecins conventionnés et les caissesmaladie obligatoires. Mais les deux restrictions mises sur les ressources financières et le nombre de médecins installés font face à une troisième dynamique qui n'est pratiquement soumise à aucune restriction: le recours des patients aux prestations médicales. Ces derniers bénéficient en effet du libre choix du médecin et ne sont soumis à aucune franchise. L'Allemagne ne prévoit pas non plus de régulation des patients par les médecins de famille. 
La question centrale qui se pose dans un système de soins budgétisés est donc la suivante: comment le droit quasi illimité des assurés aux prestations et l'accès en grande partie incontrôlé aux soins ambulatoires sontils conciliables avec le plafonnement des coûts? Cette question demeure pour l'instant sans réponse et il est évident que le monde politique rechigne à la traiter de manière sérieuse et approfondie. La première tentative de pilotage au moyen d'un supplément payé par le patient (la «taxe cabinet médical» de 10 euros par trimestre) a été abandonnée quelques années seulement après son introduction pour des motifs politiques et en raison d'erreurs dans la systématique de base. Aucune autre tentative n'a eu lieu depuis pour y répondre. Les médecins conventionnés ont proposé un modèle de tarif optionnel, lequel prévoit un pilotage par le médecin de famille, facultatif au début et obligatoire par la suite. Jusqu'à présent, cette proposition n'a pas trouvé de soutien auprès des politiques.

\section{Conséquences concrètes de la budgétisa- tion: les médecins répondent des promesses des politiques}

Par conséquent, rien n'a changé en Allemagne: avec le principe du libre choix du médecin et l'accès illimité des patients aux prestations médicales, on observe une aug- mentation permanente des contacts entre médecin et patient (environ 1 milliard en 2017) et des cas traités (environ 600 millions en 2017). En même temps, les prestations ne sont remboursées que dans le cadre du plafond budgétaire, donc conformément aux quotas fixés. Cela signifie en clair qu'un euro facturé équivaut à 90 ou au pire à 75 centimes sur le compte du médecin.

La part de travail rémunéré uniquement au prorata varie d'une région et d'un groupe de spécialistes à l'autre. Dans certains districts des associations de médecins conventionnés, le volume du budget couvre quasiment le nombre de prestations demandées. C'est le cas pour le domaine de la médecine de famille de quelques (mais de loin pas toutes) associations de médecins conventionnés en milieu rural. Dans certaines villes-Etats, par contre, les quotas ${ }^{1}$ pour les médecins de famille sont de $25 \%$, mais c'est surtout dans les domaines spécialisés que les quotas sont élevés.

Qu'est-ce que cela signifie pour la pratique individuelle? Chaque médecin doit en premier lieu décider s'il accepte de vérifier en permanence le budget «utilisé» au cours du trimestre ou s'il préfère s'exposer à la menace d'un quota sur ses prestations. Certains confrères optent pour la deuxième solution, mais nombreux sont ceux qui relèvent précisément le moment à partir duquel ils traitent leurs patients en quelque sorte gratuitement sur le plan comptable.
1 Par "quota», on entend la réduction de la rémunération de la prestation médicale sur la base du budget fixé.

\section{Comment faire avec la budgétisation? Les perspectives collective et individuelle}

Collectivement, le montant du budget et son évolution sont centraux dans un monde budgétisé. Ces deux aspects dépendent directement des ressources que la société est prête à allouer aux soins de santé et relèvent donc d'une décision politique. Le budget initial est fixé en fonction du niveau de dépenses antérieur. La systématique de sa mise à jour doit cependant répondre à la question de savoir si la société est prête à I'avenir à consacrer la même part ou une part plus élevée de ses ressources aux soins de santé.

Si cette part reste stable, le budget ne pourra augmenter qu'en fonction des revenus générés par l'assurance-maladie. Or comme la demande en prestations médicales augmente généralement plus rapidement que les recettes dans une société vieillissante, il en résulte que collectivement, les médecins ne son plus rémunérés pour toutes leurs prestations. En revanche, si l'évolution des coûts et de la morbidité entre en ligne de compte dans l'évolution du budget, il serait également nécessaire d'augmenter la part du revenu attribué aux soins de santé. Cela exige cependant aussi des prises de décision politiques.

Au niveau individuel du médecin, les conséquences dramatiques de l'introduction d'un budget collectif sont évidentes. Prenons l'exemple du dilemme du prisonnier exposé dans la théorie des jeux, rien que le concept est déjà de mauvais augure. Dans ce dilemme, chaque médecin est pris dans un système de budget collectif:

- Je sais que la somme d'argent allouée ne suffit pas à couvrir toutes les prestations, ce qui fait que seule une partie de mes prestations est rémunérée.

- Je sais que mes confrères le savent aussi. Si eux et moi, nous fournissions seulement $95 \%$ de la totalité des prestations, le montant alloué serait suffisant pour couvrir toutes les prestations.

- Or je ne sais pas comment mes confrères vont se comporter. La seule chose que je sais, c'est que celui qui fournit plus de prestations obtient plus d'argent.

Malheureusement, le dilemme du prisonnier a pour conséquence que chacun fournit le plus de prestations possibles, car $c^{\prime}$ est ainsi que les individus rationnels se comportent dans une telle situation, même s'il n'y a pas plus d'argent à disposition. Dans un tel monde, les médecins se comportent en cannibales et chaque confrère devient un adversaire dans la lutte pour la répartition du budget.

La seule solution pour éviter cette situation consiste à décomposer le budget collectif en petits budgets individuels jusqu'à l'échelle des cabinets médicaux («volume de prestations admises»), une tâche qui n'a pas été réglée de manière satisfaisante en Allemagne depuis l'introduction de la budgétisation. Les discussions autour de la répartition et des parts de budgets - inévitables dans un budget collectif - ne permettent pas d'aboutir à une solution correcte, mais seulement à des ébauches de solutions temporairement acceptables, autrement dit à des résultats de répartition acceptés au niveau politique. 
Ce sont les associations de médecins conventionnés qui déterminent quand ce point est atteint en fixant les volumes des prestations admises (Regelleistungsvolumen). Ces volumes de prestations admises définissent pour chaque cabinet un plafond régional par spécialisation. Une fois qu'il a été dépassé, les prestations peuvent être facturées, mais ne seront rémunérées qu'au prorata. Il en est de même pour les budgets des médicaments et des moyens auxiliaires (qui, en Allemagne, comprennent également la prescription de séances de physiothérapie).

\section{Situation en 2018: heures supplémentaires prescrites par l'Etat sans compensation financière?}

Depuis les années 1990, le secteur ambulatoire a subi d'importants changements. En plus de l'augmentation des maladies chroniques et du nombre de patients polymorbides combinée au vieillissement de la population, le corps médical a lui aussi changé. La fameuse pléthore médicale s'est transformée en pénurie ou plus précisément en un manque de temps de travail disponible par médecin. A cela s'ajoute que les jeunes médecins ne sont plus prêts à travailler 50 à 60 heures par semaine et qu'un plus grand nombre de médecins travaille en qualité de salariés et à temps partiel. Ainsi moins de médecins doivent fournir plus de prestations budgétisées.

Parallèlement, on assiste à un changement de mentalité au sein de la population. Le principe du libre choix du médecin est devenu une revendication du «tout, tout de suite» avec, par conséquent, la réalisation incontrôlée de doubles examens selon le principe omniprésent de consommation. Aujourd'hui, de nombreux patients veulent tout et tout de suite, ce qui se traduit par une surcharge importante des services d'urgence de certaines cliniques où les patients viennent soigner en urgence des maux connus de longue date. Ce changement culturel a été un sujet important, largement repris en le positivant au cours de la campagne électorale de 2017. Le Parti social-démocrate (SPD) avait marqué la campagne par l'expression "médecine à deux vitesses» et exigé non seulement une attribution plus rapide des rendezvous pour les patients des assurances publiques, mais également une modification en profondeur du système d'assurance en faveur d'une «assurance citoyenne» uniforme pour tous.

Aujourd'hui, l'accord de coalition du nouveau Gouvernement fédéral n'a pas repris l'assurance citoyenne. Par contre, il stipule que les médecins conventionnés doivent proposer cinq heures de consultation hebdoma- daires supplémentaires alors que leur horaire moyen est déjà de 50 heures. Une inscription dans la loi est attendue en cours d'année. Ce n'est pas seulement une intervention scandaleuse dans la liberté professionnelle des médecins conventionnés allemands et une intervention massive dans leur autonomie mais en plus, cela révèle une extension du principe budgétaire, inadmissible pour les médecins conventionnés. Cela signifie un surplus de travail imposé par l'Etat sans compensation financière.

Par conséquent, la politique de l'Union fédérale des associations de médecins conventionnés consistera ces prochains mois à utiliser cette exigence de l'accord de coalition pour rompre avec le principe de la budgétisation. Les médecins conventionnés s'engagent de manière constructive dans le processus législatif avec des propositions concrètes sur les prestations à exclure du budget. C'est absolument clair pour l'Union fédérale mais aussi pour les associations régionales, que la budgétisation doit disparaître, dans toutes ses facettes. Dans ce contexte, les propositions actuelles pour mettre un terme à la budgétisation des prestations de base ne sont que le début de son élimination progressive. En effet, la budgétisation a peut-être fait ses preuves à court terme, à un moment où un nombre artificiellement restreint de médecins étaient confinés à un budget limité pour freiner l'augmentation des coûts. Mais aujourd'hui, la situation est totalement différente. L'instrument de la planification budgétaire présente un effet contre-productif parce qu'il fait peur aux jeunes médecins qui veulent s'installer. Il doit donc être supprimé à la faveur d'une régulation responsable des patients comme c'est déjà en grande partie le cas en Suisse.

\section{L'exemple de la radiologie}

Quel devrait être le montant du budget d'un radiologue? Y-a-t'il une différence s'il utilise des procédés d'imagerie conventionnelle ou des tomodensitométries ou des IRM? Quel rôle joue la proportion de patients dont le diagnostic nécessite une radiographie conventionnelle ou un diagnostic assisté par tomodensitométrie et/ou IRM? Comment gérer le fait que le patient est référé au radiologue et, par conséquent, qu'il n'a que peu d'influence sur la taille et le type du collectif de patients? Quel budget est alloué aux institutions radiologiques coopératives? Est-ce le nombre de radiologues ou d'appareils qui est déterminant?

Des questions similaires se posent pour d'autres groupes de médecins: le budget doit-il s'adapter à l'activité individuelle ou inversement? Et qu'en est-il de la liberté thérapeutique et de la spécialisation? Qui répond à ces questions? Qui représente qui face aux intérêts des différents groupes de médecins dans le découpage du budget? 